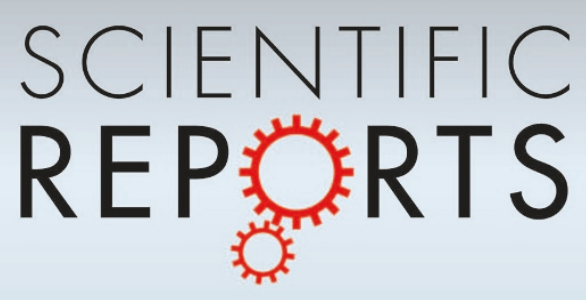

DOI: 10.1038/srep05140

\section{SUBJECT AREAS:}

PROJECTION AND

PREDICTION

ATMOSPHERIC DYNAMICS

SCIENTIFIC REPORTS:

$3: 4451$

DOI: $10.1038 /$ srep04451

(2013)

Published:

25 March 2014

Updated:

15 July 2014

\section{CORRIGENDUM: On the mechanisms of the recurvature of super typhoon Megi}

Shiqiu Peng, Yu-Kun Qian, Zhijuan Lai, Sai Hao, Shumin Chen, Hongxiong Xu, Dongxiao Wang, Xiangde Xu, Johnny C. L. Chan, Hao Zhou \& Duanling Liu

This Article contains an error in the affiliations of the authors Sai Hao, Hongxiong Xu and Xiangde Xu. The correct affiliations for these authors are listed below:

Sai $\mathrm{Hao}^{2}$, Hongxiong $\mathrm{Xu}^{3}$, Xiangde $\mathrm{Xu}^{3}$

${ }^{2}$ State Key Laboratory of Numerical Modeling for Atmospheric Sciences and Geophysical Fluid Dynamics, Institute of Atmospheric Physics, Chinese Academy of Sciences, Beijing 100029, China

${ }^{3}$ State Key Laboratory of Severe Weather, Chinese Academy of Meteorological Sciences, Beijing 100081, China; 